\title{
How can dentistry get back to work safely?
}

\author{
Parthasarathy Madurantakam
}

\section{A Commentary on}

Ather A, Patel B, Ruparel N B, Diogenes A, Hargreaves K M.

Coronavirus Disease 19 (COVID-19): Implications for Clinical Dental

Care. J Endod 2020; 46: 584-595. DOI: 10.1016/j.joen.2020.03.008.

\begin{abstract}
Data sources Narrative review

Study abstract This review provides a synopsis of our understanding of severe acute respiratory syndrome coronavirus 2 (SARS-CoV-2) and its associated coronavirus disease, COVID-19. The virus is shed in the nasopharyngeal and salivary secretions of carriers, and this puts dental professionals at risk for increased exposure of SARS-CoV-2. The paper summarises the current guidelines outlined by the CDC and presents the triaging protocols to identify potential carriers and how to safely limit treatment to low-risk patients.
\end{abstract}

\section{Commentary}

The publication by Ather et al. is a comprehensive overview of our current understanding (which changes by the day) of SARSCoV 2 pandemic. The article addresses important aspects of clinical dentistry affecting dental practitioners as they consider reopening their offices for elective procedures after the COVID pandemic.

The article is a useful reference for patients, staff and dentists regarding the roles that each need to play to be able to safely receive/provide dental care post-COVID. The importance of asking screening questions before scheduling an appointment, and again in the waiting area forms the first line of defence for avoiding a COVID carrier being in the dental chair. Subjective reporting

\section{Practice point}

- With information coming out from so many different sources, it is important to have a reliable resource that is consistently updated as we learn more about this virus

- On line updates are likely to offer more up to the minute information than print media

- It is highly recommended that guidelines are followed given that most elective dental procedures generate high amounts of aerosols and a significant number of patients could be asymptomatic carriers

- Such recommendations can be expected to become 'routine' in the future - at least until accurate testing and effective vaccination become common place. ${ }^{1}$

with questionnaires coupled with objective measurement of temperature in the waiting area may do much to mitigate the risk of COVID spread within and/or from the dental office.

The clinical protocols for identifying emergency situations (and its management) as well as appropriate triaging are well detailed in the article. The decision flow charts make it easy to read and comprehend. The references regarding appropriate personal protective equipment (PPE) are from the Centres for Disease Control and Prevention (CDC).

The main limitation of this paper is that most of the recommendations are drawn from $\mathrm{CDC}$ and the ADA that are based in the US. It is important to emphasise that practitioners from other regions of the world would need to refer to their own government organisations for specific guidelines.

\section{Author affiliation}

VCU School of Dentistry, Lyons Building 415, 520 N. 12th Street, Richmond, VA 23,298, USA

\section{Reference}

1. Proffitt E. What will be the new normal for the dental industry? Br Dent / 2020; 228: 678-680.

Evidence-Based Dentistry (2020) 21, 48.

https://doi.org/ 10.1038/s41432-020-0090-x 